\title{
Emofiltrazione arterovenosa continua e tecniche derivate
}

\author{
M. Milan, C. Ronco \\ Divisione di Nefrologia, Ospedale San Bortolo - Vicenza
}

1 decorso dell'insufficienza renale acuta (IRA) trattata con emodialisi giornaliera od intermittente è generalmente favorevole, e presenta una elevata percentuale di ripresa funzionale. Tuttavia, nei pazienti in terapia intensiva I'IRA viene frequentemente complicata da patologie concomitanti e disfunzioni multiorganiche, che comportano una prognosi più severa e possono rappresentare ulteriori fattori di rischio o controindicazioni al trattamento dialitico extracorporeo o peritoneale (2-4).

Allo scopo di superare tali problemi, Kramer e Coll. (5) nel 1977 descrissero una nuova metodica denominata EMOFILTRAZIONE ARTERO-VENOSA CONTINUA (CAVH), proposta come forma di terapia sostitutiva alternativa all'emodialisi intermittente.

L'applicazione clinica della CAVH rimase limitata ad un gruppo ristretto di ricercatori fino al 1983, quando Lauer A et al (6) descrissero le singolari caratteristiche operative del sistema e ne delinearono il potenziale impatto nel trattamento dell'IRA nei reparti di terapia intensiva.

La CAVH è un trattamento extracorporeo in cui l'acqua, gli elettroliti ed altri soluti di basso peso molecolare vengono rimossi dal paziente mediante convezione in un periodo di tempo prolungato. L'ultrafiltrazione ha luogo in un filtro di piccole dimensioni grazie al gradiente pressorio generato ai due lati di una membrana semipermeabile $(7,8)$. Contemporaneamente, il volume circolante viene ricostituito mediante reinfusione di fluidi di sostituzione di composizione chimica simile a quella dell'acqua plasmatica. Non è necessario l'impiego di pompe peristaltiche, poiché il sangue procede nel circuito extracorporeo spinto dal gradiente pressorio arterovenoso. L'obbiettivo è quello di ottenere una quantità di ultrafiltrato di 12-18 litri nelle 24 ore, che rappresenta l'equivalente di una filtrazione glomerulare di $8-14 \mathrm{ml} / \mathrm{minuto}$.

L'ultrafiltrato può essere rimpiazzato completamente, allo scopo di ridurre la concentrazione dei soluti plasmatici, o parzialmente, qualora l'obbiettivo sia quello di modulare la concentrazione di soluti ed il volume circolante; oppure può non essere sostituito, per ottenere esclusivamente un controllo del bilancio di fluidi (9).

La CAVH è stata ampiamente utilizzata in questi ultimi anni per il trattamento del sovraccarico di fluidi refrattario alla terapia diuretica, dell'IRA complicata, degli squilibri idroelettrolitici e delle alterazioni dell'equilibrio acido-base, nonché quale terapia alternativa in pazienti critici, in cui la dialisi tradizionale presenta gravi problemi di ordine clinico e tecnico. La metodica si è dimostrata valida soprattutto per la semplicità e facilità di monitoraggio e per l'elevato grado di tollerabilità clinica, trovando tuttavia i suoi maggiori limiti per una più ampia applicazione clinica, nella bassa efficienza del sistema e nella facile coagulazione del filtro. Lo sviluppo di nuovi materiali e nuove tecniche ha recentemente permesso di superare questi problemi. D'altra parte, l'uso sempre più frequente di piccole pompe peristaltiche in circolazione veno-venosa (CVVH) ha consentito una buona standardizzazione della procedura, eliminando i rischi connessi alle manovre di incannulazione arteriosa. Questi progressi hanno quindi risvegliato un nuovo interesse per i trattamenti sostitutivi continui dell'IRA, che sono oggi sempre più frequentemente impiegati nelle diverse situazioni cliniche.

\section{Accesso vascolare}

Sia l'emofiltrazione che l'emodialisi continua possono essere effettuate in circolazione spontanea artero-venosa (CAVH-CAVHD) o in circolazione veno-venosa servoassistita da pompa (CVVH - CVVHD).

In circolazione artero-venosa l'accesso vascolare deve garantire un gradiente artero-venoso tale da consentire un adeguato flusso ematico exracorporeo (10, 11). L'incannulazione percutanea dell'arteria e della vena femorali fornisce un accesso adattato per la CAVH, ma 
possono essere vantaggiosamente utilizzate anche l'arteria brachiale e la vena basilica o cefalica, la giugulare o la succlavia.

I cateteri devono avere un lume di almeno $2 \mathrm{~mm}$, e la loro lunghezza non deve essere superiore agli $80-100 \mathrm{~mm}$, onde garantire una bassa resistenza al flusso: per lo stesso motivo, nel circuito extracorporeo devono essere previste linee corte e connessioni adeguate.

In alcuni pazienti può essere utilizzato to shunt di Scribner; tuttavia, questo tipo di accesso è facilmente soggetto ad occlusione per coagulazione, rendendo inoltre i vasi inutilizzabili per l'eventuale successivo allestimento di una fistola arterovenosa.

L'emofiltrazione veno-venosa continua (CVVH) e l'emodialisi veno-venosa continua (CVVHD) sono sempre più utilizzate nella pratica clinica, inserendo nel circuito una pompa peristaltica. In questo caso vengono incannulati due vasi venosi periferici, oppure una singola vena con un catetere a doppio lume.

Qualora si usi una pompa a doppia testata è possibile utilizzare anche un catetere venoso a lume singolo.

L'uso della pompa potrebbe ridurre parzialmente i vantaggi del trattamento artero-venoso in termini di stabilità vascolare, rischio di embolia gassosa ed auto-limitazione dell'ultrafiltrazione; d'altra parte consente di eseguire il trattamento anche in pazienti con scarso gradiente pressorio artero-venoso o con controindicazioni all incannulamento arterioso.

Operativamente, con pressioni arteriose medie di $60-80 \mathrm{mmHg}$, in CAVH si ottengono flussi ematici da 50 a 120 millilitri/minuto. In CVVH il flusso ematico può essere modulato a valori anche superiori senza particolari problemi tecnici (12).

Si è molto discusso circa i vantaggi delI'impiego dell'uno o dell'altro sistema. Noi pensiamo che entrambi gli approcci dovrebbero essere presi in considerazione, in rapporto alle condizioni cliniche del paziente, alle disponibilità tecniche, al grado di addestramento del personale infermieristico ed all'esperienza dello staff medico.

\section{Circuito extracorporeo}

In CAVH il flusso ematico e, conseguen- temente. l'ultrafiltrazione possono essere influenzati da vari fattori $(13,14)$.

$\mathrm{Nel}$ circuito ematico possono essere schematizzati cinque siti di resistenza al flusso: l'accesso arterioso, la linea arteriosa, l'emofiltro, la linea venosa e l'accesso venoso.

Le linee arteriosa e venosa dovrebbero essere quanto più brevi possibile, onde evitare cadute pressorie lungo il circuito. La bassa resistenza del circuito permette di incrementare il flusso ematico e la velocità di ultrafiltrazione ad un dato gradiente pressorio artero-venoso.

L'impiego di una pompa ematica rende tuttavia irrilevante l'importanza di questi fattori, poiché la costanza del flusso ematico è servocontrollata.

\section{Membrane, ultrafiltrazione e clearance}

In CAVH vengono usualmente impiegate membrane ad alta permeabilità, al fine di ottenere ultrafiltrazioni e clearance convettive dei soluti adeguate (15). In queste membrane i soluti vengono rimossi per convezione, grazie ad un effetto di solvent-drag. La permeabilità delle membrane condiziona il passaggio delle molecole (il coefficiente di riflessione di Stavermann $(\sigma)$ è una caratteristica intrinseca della membrana, che implica un determinato coefficiente di sieving osservato (s)). Se il soluto non viene respinto dalla membrana ( $s=1)$, il rapporto tra la concentrazione nell'ultrafiltrato (UF) e la concentrazione plasmatica (P) è uguale ad 1, e la clearance $(\mathrm{K})$ corrisponde alla velocità di ultrafiltrazione (Qf).

$(\mathrm{K}=\mathrm{Qf} *[\mathrm{UF}] /[\mathrm{P}]=\mathrm{Qf} * 1=\mathrm{Qf})$

Qf è proporzionale al prodotto tra coefficiente di permeabilità della membrana e pressione di transmembrana (Kf* TMP): la pressione di transmembrana (TMP) è calcolata con la formula:

$\mathrm{TMP}=\mathrm{Pb}+\mathrm{Puf}-\pi$

dove $\mathrm{Pb}$ è la pressione idrostatica del sangue e Puf è la pressione negativa esercitata dalla colonna di ultrafiltrato e $\pi$ è la pressione oncotica plasmatica. La pressione idrostatica $(\mathrm{Pb})$ sarebbe insufficiente per ottenere significativi volumi di ultrafiltrato senza il contributo della pressione negativa Puf. Per questo motivo per ottimizzare il rendimento del sistema è di importanza critica collocare la sacca di raccolta dell'ultrafiltrato nella posizione più declive possibile rispetto al filtro.

L'applicazione di una ulteriore pressione negativa, con marcata suzione nel comparto dell'ultrafiltrato non è raccomandabile, poiché favorirebbe fenomeni di coagulazione del filtro, in relazione con eccessive frazioni di filtrazione.

Le membrane cellulosiche più sottili, come il Cuprofan, non sono dotate di sufficiente permeabilita idraulica per garantire un'adeguata rimozione di fluidi ai regimi pressori operanti in CAVH. Queste membrane sono in grado però di offrire una buona rimozione di soluti di basso peso molecolare per via diffusiva. Esse pertanto sono preferibilmente impiegate in emodialisi arterio-venosa continua (CAVHD), allo scopo di rimuovere maggiori quantità di urea. In questo caso la diffusione viene facilitata dal ridotto spessore della membrana, dall'elevato coefficiente di diffusione dell'urea (soluto di basso peso molecolare), e dal gradiente di concentrazione esistente tra sangue e liquido di dialisi (il flusso del liquido di dialisi (Qd) viene generalmente mantenuto tra 10 e $40 \mathrm{ml} / \mathrm{min}$ ). In CAVHD, quando si raggiunge il completo equilibrio dei soluti all'interno del dializzatore, le clearance corrispondono al flusso del dialisato in uscita (equazione 3$)$.

$\mathrm{K}=\mathrm{Qd} *[\mathrm{D}] /[\mathrm{P}]=\mathrm{Qd} * 1=\mathrm{Qd}$

Se i soluti non si equilibrano completamente i valori di clearance calcolati dal lato del dialisato corrispondono al prodotto del flusso del dialisato per il rapporto tra estrazione di soluti al minuto e concentrazione del soluto nel sangue all'ingresso del filtro (equazione 4).

$\mathrm{K}=\mathrm{Qd} *[\mathrm{D}] /[\mathrm{P}]$

I soluti di maggior peso molecolare hanno coefficienti di diffusione inferiori ed un ingombro sterico maggiore, per cui vengono respinti dalle membrane di dialisi. La convenzione e l'uso di membrane con coefficienti di permeabilità più elevati divengono quindi fondamentali 
per la rimozione di tali molecole (15), Nei trattamenti misti, in cui vengono combinate diffusione e convezione, è richiesto l'impiego di polimeri con caratteristiche intermedie, per cui vengono utilizzate membrane in poliacrilonitrile o membrane sottili in polisulfone. Questo compromesso tra porosità e spessore della membrana consente di ottenere una efficace rimozione di soluti in un ampio range di pesi molecolari.

\section{Emofiltri ed emodiafiltri}

La CAVH è un sistema che opera a bassi regimi di flusso ematico e di pressioni ed in condizioni di equilibrio di pressione di filtrazione (14, 16-18). Pertanto gli emofiltri devono essere costruiti con dimensioni e geometrie idonee a tali condizioni operative.

Negli emofiltri a fibre cave, man mano che l'acqua plasmatica viene rimossa per ultrafiltrazione, aumentano la concentrazione proteica plasmatica, l'ematocrito e la viscosità. La progressiva diminuzione della pressione idrostatica del sangue si accompagna quindi ad un progressivo aumento della pressione oncotica generata dalle proteine: esiste pertanto un punto, lungo il filtro, in cui pressione idrostatica e pressione oncotica, agenti in direzioni opposte, si equilibrano. Da questo punto in poi, aumentano la resistenza al flusso ed il rischio di coagulazione, mentre la filtrazione cessa.

In base a queste osservazioni sono stati modificati il disegno e la geometria di flusso degli emofiltri per CAVH (16).

Secondo la legge di Hagen-Poiseuille, un filtro più corto $\mathrm{e}$ di maggior sezione trasversa permette di evitare il raggiungimento dell'equilibrio di pressione di filtrazione, grazie ai maggiori flussi ematici ottenibili per un dato gradiente artero-venoso. I risultati ottenuti con i nuovi filtri hanno confermato questa ipotesi ed hanno permesso di ridurre la richiesta di eparina, pur aumentando la vita media del filtro (16, 17).

Sono state sviluppate anche nuove fibre con maggior diametro interno ( $250 \mathrm{mi}$ cra), in grado di garantire una minor resistenza e di ridurre quindi il rischio di coagulazione nel segmento terminale del filtro (16).

In alternativa sono disponibili per
CAVH anche filtri a piastre parallele. Nonostante l'ingombro dell involucro di questi emofiltri. la geometria a piastra può rappresentare un reale vantaggio riducendo la richiesta di eparina durante il trattamento (19). In ogni caso comunque, tutti questi accorgimenti tecnici divengono di secondaria importanza quando nel circuito sia inserita una pompa ematica che controlla il flusso extracorporeo indipendentemente dalla pressione arteriosa del paziente.

Dato il sempre più frequente impiego dell'emodialisi artero-venosa e veno-venosa continua, tutti i filtri sono inoltre ora dotati di una doppia porta per la circolazione del liquido di dialisi.

Sono ormai disponibili diversi tipi di emofiltri per trattamenti extracorporei continui, commercializzati da diverse ditte. Qualora si utilizzi una pompa ematica o comunque in pazienti con pressione arteriosa stabile, possono essere impiegati filtri a superficie relativamente ampia $(0.6 \mathrm{mq})$; filtri più corti e di superficie minore $(0.2 \mathrm{mq})$, a bassa resistenza, possono essere invece molto utili in pazienti gravemente ipotesi, in cui si può prevedere uno scarso flusso extra- corporeo. Una scelta così orientata consente maggior durata del filtro con somministrazione di minori quantità di eparina, riducendo l'incidenza di interruzioni del trattamento per cause tecniche $(20,21)$.

\section{Efficienza e qualità del trattamento}

Per ottenere un buon controllo metabolico dell'IRA con la CAVH, è necessaria una ultrafiltrazione di almeno 12-16 litri al giorno. Quando la metodica classica non consente il raggiungimento di questo obiettivo, può essere necessario ricorrere alla prediluizione, all applicazione di pressione negativa sull'ultrafiltrato, alla circolazione in pompa, all'applicazione aggiunta della componente diffusiva o di altre tecniche, onde garantire una adeguata depurazione ematica (Fig. 1) (22-31).

La CAVH, cosi come originariamente concepita, è in grado di garantire un massimo di 17 litri $/ 24$ ore di ultrafiltrato. con puro trasporto convettivo $(30)$.

\section{CLEARANCE DELL'UREA CON TECNICHE DIVERSE}
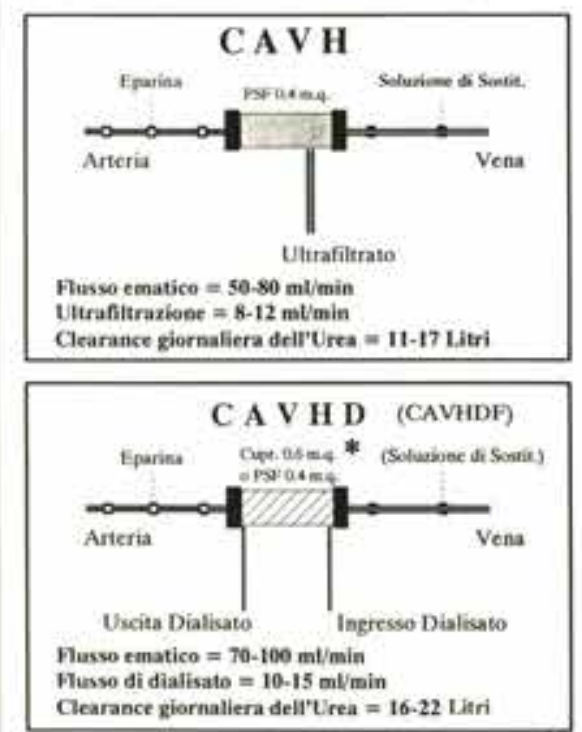

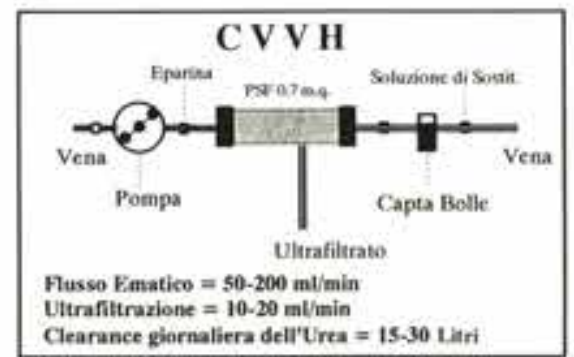

Clearanee giornaliera dell'Urea $=15-30$ Litri

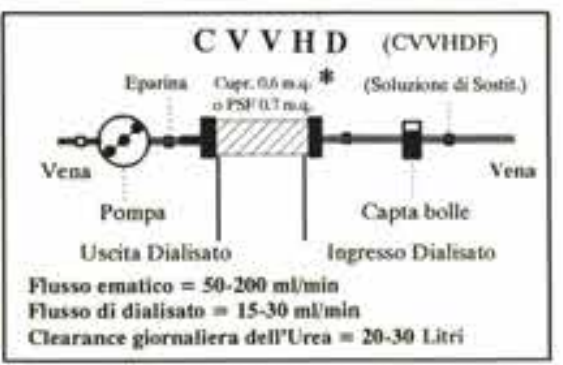

* La soluzione di wostituzione è necessaria unicamente quando si usino membrane ad alta permeabilita idraulica. In questo caso it trattamento viene chiamato emodiafiltrazione continua (CAVHDF - CVVHDF)

Fig. 1. Possibill tecniche per aumentare lefficienza dei trattamenti continui. La clearance dell arca può essere incrementata aumentando f'ulmafiltrazione in emofiltrazione con pompa, o aggiungendo ana componente diffusiva came nell emodialist artero-venosa o veno-venosa cantimu. In tal caso, quamio si impiegano nucmbrane ad alra permeabilita, si raggiumgono elevati volumi di ultrafiltrato che inpongono $T$ ieso di reinfusiomi per il mantenimento del bilancio idrica. Questo tipo di trattamento sarebbe pia propriamente denominato emadiafiltrassione, Quando si impiegano membrane a bassa permeabilità non ż necessario effernare reinfasione, ed il tranamento pus essere denamisato emodialisi cominina. 
Nuovi emofiltri, ad alto rendimento, possono giungere ad un totale di $20-22$ litri/24 ore, che tuttavia possono risultare non sufficienti. L impiego di una pompa consente facilmente di aumentare il flusso ematico e di raggiungere clearance sino a 30 litri/24 ore. In questi casi tuttavia si rende necessario provvedere alla reinfusione di grandi quantita di liquido, e ciò rende più difficile un corretto bilancio di fluridi.

Molti Autori hanno descritto la possibilità di usare un sistema misto diffusivoconvettivo (30-35) od un sistema puramente diffusivo (33) per il trattamento dell'insufficienza renale acuta in pazienti critici. In questo caso comunque, pur ottenendosi generalmente soddisfacenti clearance delle piccole molecole, la clearance delle medie molecole può essere considerevolmente ridotta. Poiché nei pazienti in terapia intensiva con IRA. sepsi, insufficienza multiorganica e grave catabolismo, possono essere presenti elevati livelli di sostanze di medio peso molecolare (500-5000 Daltons) come mediatori chimici, sostanze vasoattive, citochine (TNF, Interleuchina 1, PAF ecc.), un adeguato trattamento dovrebbe prevedere non solo la rimozione del
BUN, ma anche una rimozione efficace di queste sostanze. In queste situazioni la necessaria clearance convettiva può essere ottenuta solamente con l'impiego di membrane sintetiche ad alti flussi, do. tate di elevati coefficienti di sieving.

Per raggiungere un compromesso, mantenendo il requisito di adeguato rapporto fra quota di convezione e diffusione, di ridotti volumi di reinfusione e di facile monitoraggio, abbiamo impiegato un sistema denominato DIALISI CONTINUA AD ALTO FLUSSO (CHFD) (Figura 2) (36). Il sistema consiste in un circuito per emodialisi continua, modificato allo scopo di ottenere un controllo in continuo del volume di dialisato. Viene utilizzato un emodiafiltro con membrana ad alta permeabilità di $0.6 \mathrm{mq}$ di superficie (D-30 AMICON Danvers Mass. USA, FH66 GAMBRO LUND Svezia, Multiflow 60 HOSPAL Lione Francia), e nel circuito del liquido di dialisi vengono inserite due pompe peristaltiche. Il flusso in entrata del liquido di dialisi è controllato dalla prima pompa (P1). La seconda pompa (P2), applicata sulla linea di uscita, regola l'ultrafiltrazione netta secondo uno specifico modulo di programmazione. II sistema puó operare in condizioni di passo sin-

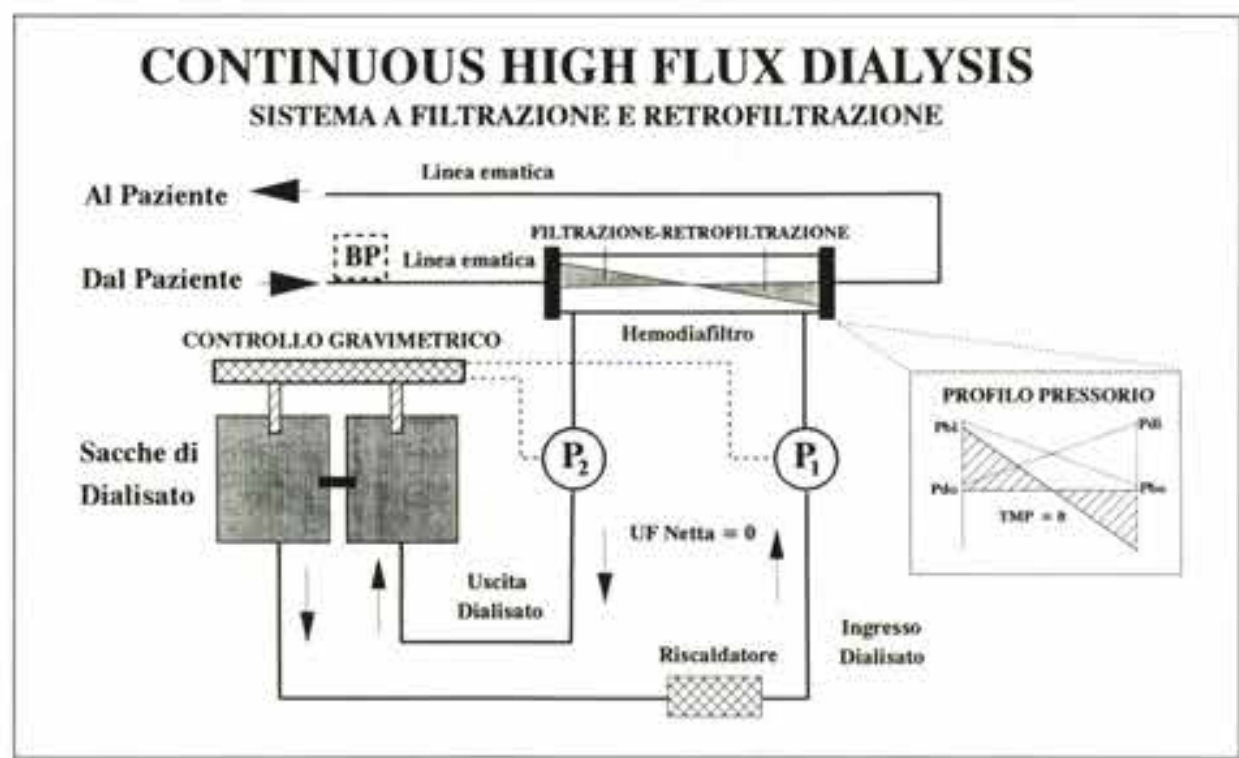

Fig. 2 - Rappresentazione schematica della dialisi contimia ad alto flusso (CHFD). Vengono ricircolati $10 \mathrm{~h}$ tri di liquido di dialisi sterile apirogeno con bicarbonato ad un flusso di $100 \mathrm{ml} / \mathrm{min}$. L'ultrafiltrazione pab essere programmata e controllata in base alla diversa velocità delle due pompe (P) e P2), Un sistema di controllo grovimetrico, che controlla P2, garantisce la precisione del bilancio di fluidi. L'alta permeabilité del. Temodiafiltro ed il particalare regime pressorio operativo producono il tipico profito di filtrazione netta sero, che stabilisce il meccanismo di fittrasione-retrofittratione, In questo caso, realit:andosi I ultrafiltratione sempre nel segmento prossimale del filtro, viene controllato il bilancio di fluidi e preservato il trasporto convettivo, assicurando una rimozione di grosse molecole maggiore di quella ottenibile con F emodialisi. Le sacche vengono cambiate ogni 4 ore, quando la concentrasione di urea nel liquido si re equilibrata con quella del sangare.

golo o di ricircolo del liquido di dialisi. Nella modalità a ricircolo la stessa quantità di liquido di dialisi può essere sfruttata al meglio, riducendo il costo del trattamento.

In questo sistema, una volta raggiunto il peso secco del paziente, il circuito può continuare ad operare ad ultrafiltrazione netta zero, utilizzando un meccanismo di filtrazione e retrofiltrazione di liquido di dialisi sterile a flussi di $50-150 \mathrm{ml} / \mathrm{min}$. Come descritto in Figura 3, 1'equilibrazione tra dialisato e plasma per l'urea e la creatinina viene raggiunta dopo 4 ore di trattamento utilizzando un pool ricircolante di dialisato di 10 litri.

$\mathrm{E}$ interessante notare che, allo stesso tempo di trattamento, il rapporto dialisato/plasma per molecole più grandi, come l'inulina, è di 0.6. Con un trattamento continuo in cui le sacche di liquido di dialisi vengano cambiate ogni 4 ore, si otterranno clearance massime di $60 \mathrm{li}$ tri/die per l'urea e di 36 litri/die per l'inulina, che rappresentano una depurazione ematica molto efficiente. Infatti in queste condizioni la clearance giomaliera è vicina, se non superiore, allo spazio totale di distribuzione dell urea della maggioranza dei pazienti. In questo caso la clearance frazionaria dell'acqua totale corporea $(\mathrm{K} / \mathrm{V})$ si avvicina o supera il valore di 1 ogni 24 ore $(t)$. Se la CFHD viene eseguita continuativamente, il $\mathrm{Kt} / \mathrm{V}$ settimanale può rientrare in un range compreso tra 7 e 10 , risultandone quindi un trattamento molto più efficiente di altre terapie dialitiche intermittenti (35). Quando non sia richiesto un tale grado di efficienza. le sacche possono essere cambiate ogni 6 ore e, mentre l'equilibrazione per l'urea sarà invariata, l'equilibrazione per le grosse molecole sarà in questo caso ancora maggiore. Non si effettua alcuna reinfusione, poiché il sistema opera ad ultrafiltrazione netta zero (filtrazione-retrofiltrazione). L'elevata clearance dell'inulina è ottenuta principalmente per trasporto convettivo, che avviene nel tratto prossimale del filtro. L'ultrafiltrazione netta di zero è infatti il risultato di un meccanismo di filtrazione nel segmento prossimale e retrofiltrazione nel segmento distale del filtro. La CHFD è pertanto un sistema simile all emodiafiltrazione, in cui l'ultrafiltrato viene prodotto nella prima metà della fibra e la reinfusione avviene nella seconda metà per retrofiltrazione di liquido di dialisi sterile. 


\section{CLEARANCE DEI SOLUTI A DIVERSI FLUSSI DI LIQUIDO DI DIALISI IN PASSO SINGOLO}

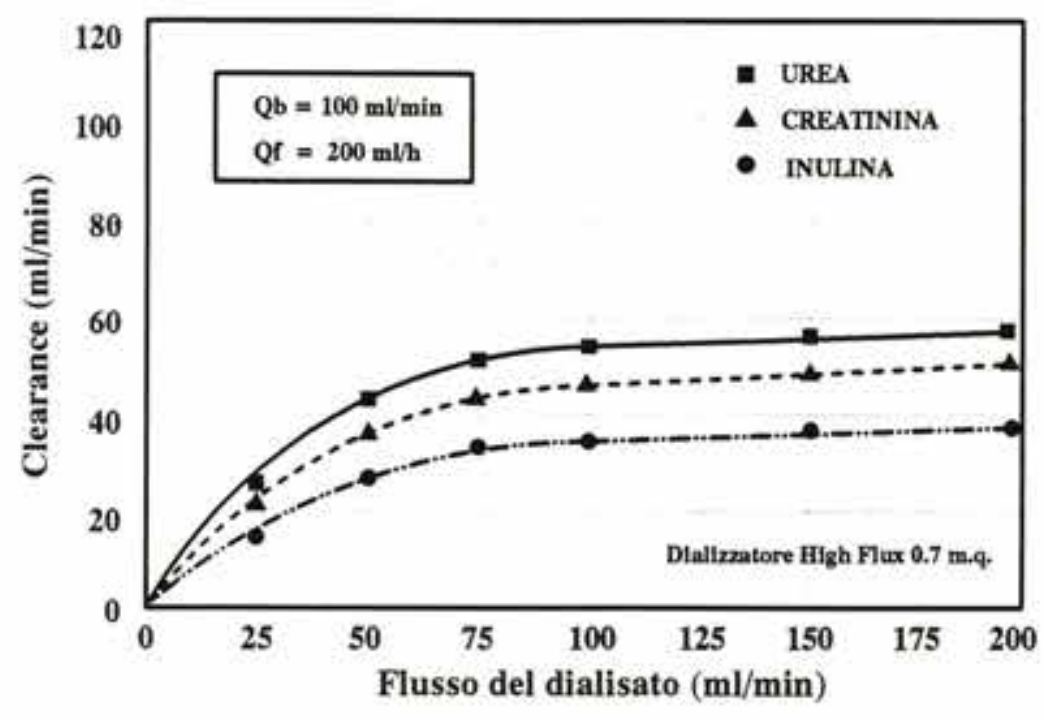

\section{EQUILIBRAZIONE DIALISATO/PLASMA IN RICIRCOLO CON FLUSSO DI DIALISATO $=100 \mathrm{ml} / \mathrm{min}$}

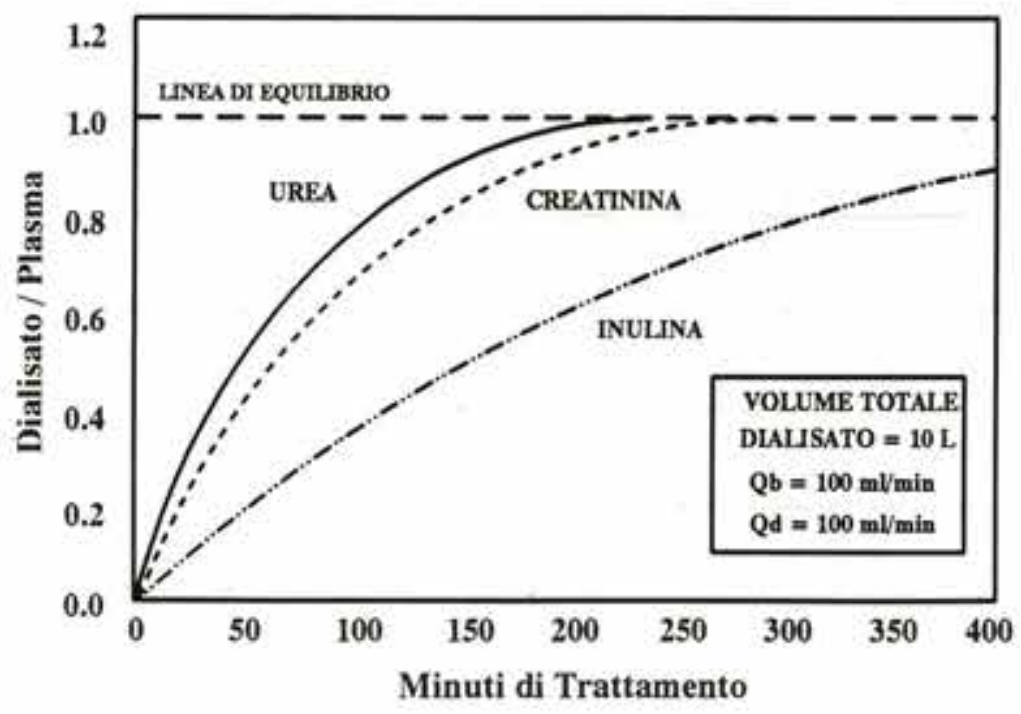

Fig. 3. Clearance dei soluti in dialisi contina ad alto fasso. Nel riquadro saperiore sono rappresentate le clearance sperimentali in passo singolo in rapporto al flusso del dialisato. II platean viene raggiunfo ad un flusso di dialisato di $100 \mathrm{ml} / \mathrm{min}$, suggerendone quindi rimpiego in ricincolo. Nel riquadro inferiore é riportata lequilibratione dialisatolplasma di vari soluti a diversi tempi di ricircolo. Alla quarta ora lurea e la creatinina si equilibrano, mentre per molecole pia grosse come linulina si dimostra un rapporto dialisatolplasma di 0.6 .

Per riassumere, mentre la CAVH o la CVVH rappresentano trattamenti convettivi e CAVHD-CVVHD sono terapie puramente diffusive, la CAVHDFCVVHDF (emodiafiltrazione continua) e CAVHDF-CVVHFD (dialisi continua ad alto flusso) rappresentano una vera $\mathrm{e}$ propria forma mista di trattamento.
Comunque, mentre nel modello operativo della emodiafiltrazione sono richiesti grandi volumi di liquido di reinfusione. nella dialisi ad alto flusso da nói impiegata il liquido di dialisi viene utilizzato sia come tale che come liquido di reinfusione. Si è molto discusso circa l'opportunità di usare liquido di dialisi con tam- pone bicarbonato piuttosto che lattato od acetato. Non si deve però dimenticare che le soluzioni con bicarbonato presentano l'inconveniente di breve durata della preparazione e che l'aggiunta di calcio deve essere effettuata al momento dell'uso, creando problemi di stoccaggio e purezza delle soluzioni. Poiché il lattato può essere impiegato con sicurezza nella maggioranza dei pazienti fornendo un 'adeguata correzione dell'acidosi metabolica, è consigliabile l'impiego di soluzioni di dialisi sterili con bicarbonato solamente nei pazienti in cui, per particolari condizioni cliniche, si suppone una scarsa capacità di metabolizzazione del lattato (per cui diviene importante il controllo dell anion gap).

\section{Anti-coagulazione}

Una anti-coagulazione efficace può essere ottenuta con infusione continua di eparina durante il trattamento $(6,40)$. L'obbiettivo ideale è il massimo effetto anticoagulante all'interno del filtro con minimi effetti sistemici soprattutto nei pazienti con elevato rischio emorragico. In CAVH questa possibilità è strettamente legata al rispetto di specifiche procedure: 1) II filtro deve essere accuratamente lavato prima dell'uso con almeno 2 litri di soluzione fisiologica eparinata. 2) II flusso ematico spontaneo ottenibile dal gradiente artero-venoso del paziente non deve essere ostacolato da resistenze superflue lungo il circuito. 3 ) Uso della tecnica di prediluizione quando necessario. 4) Frequenti lavaggi del sistema con boli di soluzione fisiologica nel filtro. 5) Uso della pompa sangue quando necessario. Un' infusione eparinica di 300-600 U.1./ora è di regola adeguata per garantire la sicurezza del trattamento (40).

L'infusione continua di eparina direttamente nella linea arteriosa del circuito extracorporeo offre il vantaggio di una certa autoregolazione dell'effetto anticoagulante $(41,42)$. Quando diminuisce il flusso ematico, infatti, la concentrazione di eparina aumenta nel circuito extracorporeo ma non nel circolo sistemico. Le membrane rivestite di eparina sono ancora allo stadio sperimentale (44). mentre sembra promettente l'impiego alternativo di eparine a basso peso molecolare e di altre sostanze anticoagulanti 
$(43,45,46)$. L'uso della pompa ematica può ridurre significativamente la richiesta di eparina grazie alla stabilità del flusso ematico attraverso il filtro. Se la velocita della pompa viene mantenuta relativamente elevata, il trattamento può essere eseguito in qualche caso anche senza eparinizzazione.

\section{Liquidi di reinfusione}

La natura dei liquidi di reinfusione varia a seconda delle necessitả cliniche del paziente. L'istituzione precoce di un programma di nutrizione parenterale totale ben bilanciato può contribuire all'ottenimento di un miglior controllo metabolico $(46.47)$.

La correzione dell'acidosi metabolica e degli squilibri elettrolitici rappresenta un altra importante indicazione per la terapia con CAVH (48).

Solo il modello dell'emofiltrazione, che riproduce la logica operativa del rene umano, può consentire una fine regolazione degli elettroliti e dei tamponi. Nei trattamenti diffusivi il bilanciamento delle diverse sostanze è meno facilmente modulabile. Nel caso della dialisi continua ad alto flusso, il liquido di reinfusione è costituito dallo stesso liquido di dialisi, e la sua composizione può essere modificata aggiungendo diversi soluti a seconda delle esigenze cliniche del paziente.

\section{Indicazioni cliniche}

Le indicazioni cliniche ai trattamenti dialitici continui non sono ancora state completamente definite. Le terapie continue comunque non possono più essere considerate come semplici alternative alla dialisi standard. 1 pazienti in terapia intensiva candidati a trattamento continuo presentano generalmente caratteristiche diverse da quelli degenti in normali reparti nefrologici. Tali pazienti si trovano in condizioni cliniche critiche, presentando una serie di complicazioni di ordine internistico e chirurgico c, data la loro grave instabilità, necessitano $\mathrm{di}$ una terapia dialitica delicata e progressiva (49). I trattamenti continui possono risultare utili in pazienti con insufficienza renale acuta complicata, associata o meno a disfunzione multiorganica, sepsi ed altre condizioni patologiche. II trattamento precoce, la possibile rimozione di mediatori chimici e l'effettiva "emoequilibrazione" data dalla continuità della terapia possono sostenere il paziente in modo determinante nella fase critica della sindrome. Per "emoequilibrazione" si deve intendere una completa correzione degli squilibri metabolici (bilancio di fluidi, bilancio elettrolitico, bilancio acido-base, nutrizione) e non esclusivamente l'aspetto relativo alla depurazione del sangue. In quest'ottica, la precoce istituzione del trattamento potrebbe contribuire alla normalizzazione delle concentrazioni di particolari sostanze (IL-1. TNF. PAF) rilasciate in circolo, che conducono il paziente verso la "zona pericolosa" della sindrome di insufficienza multiorganica. D'altra parte, essendo pur sempre una tecnica invasiva, la terapia dialitica continua comporta di per sé dei rischi che devono essere attentamente valutati in rapporto ai possibili benefici.

II sovraccarico di liquidi è di comune riscontro nei pazienti critici, con imbibizione polmonare e relativa alterazione degli scambi gassosi e con diminuito flusso plasmatico renale.

La CAVH consente una progressiva rimozione di fluidi dal paziente in assenza di significativi episodi ipotensivi e/o ulteriore peggioramento della perfusione parenchimale.

Per spiegare questa particolare stabilità cardiovascolare in $\mathrm{CAVH}$ sono stati considerati diversi fattori $(50,55)$ : la lenta e continua ultrafiltrazione, il refilling plasmatico (dovuto ad ultrafiltrazione iso-osmotica). la stabilita del sistema renina-angiotensina, la stabilità dell'osmolarita extracellulare. In particolare la $\mathrm{CAVH}$ permette la rimozione indipendente di sodio ed acqua, variando la composizione della soluzione di reinfusione (51). Per esempio. è possibile ottenere 2 litri di ultrafiltrato a concentrazione sodica di $140 \mathrm{mEq} / \mathrm{l}$, senza reinfusione. Il paziente avrà quindi un calo ponderale di 2 chilogrammi ed una perdita totale di $280 \mathrm{mEq}$ di sodio. Oppure possiamo rimuovere 10 litri di ultrafiltrato con concentrazione sodica di $140 \mathrm{mEq} /$. reinfondendo 8 litri di soluzione a concentrazione sodica di $130 \mathrm{mEq} / \mathrm{l}$. Anche in questo caso il calo ponderale sarà di 2 chilogrammi, ma la rimozione di sodio sarà di $360 \mathrm{mEq}$.

L'incremento dell'indice cardiaco e la parallela riduzione delle resistenza vascolari periferiche generalmente osservati durante trattamento con CAVH e CVVH suggeriscono l'impiego di queste metodiche nei pazienti con insufficienza renale acuta associata a miocardiopatia ed insufficienza cardiaca congestizia.

Nei pazienti con shock settico od instabilità cardiovascolare associata ad insufficienza multiorganica, la vasodilatazione e l'aumentata permeabilità vascolare sono dovute a diversi mediatori derivanti da degranulazione leucocitaria, attivazione delle cellule endoteliali e stimolazione macrofagica. In tali pazienti il miglioramento del controllo pressorio osservato durante CAVH è probabilmente correlato alla rimozione dal circolo di queste sostanze vasoattive (TNFalfa, Interleuchina-1, PAF ecc) $(56,57)$.

Nonostante la bassa efficienza depurativat. la CAVH può essere usata con successo in pazienti con IRA che non possono essere trattati con altri tipi di terapia per diversi motivi. In presenza di grave stato catabolico possono essere adottate diverse procedure di supporto per aumentare l'efficienza del sistema (58-60). Un programma di terapia parenterale di supporto può contribuire a ridurre il protein catabolic rate ed il suo effetto sulla concentrazione di BUN.

Sebbene gli emofiltri di nuova generazione consentano ultrafiltrazioni di 10 $25 \mathrm{ml} /$ minuto, talvolta la condizione di ipotensione del paziente limita la possibilità di ottenere adeguati flussi ematici e di mantenere il controllo del BUN.

In questi casi, l'emofiltrazione veno-venosa con pompa ematica, essendo indipendente dal gradiente pressorio arterovenoso del paziente, risulta generalmente più efficiente. La velocità di ultrafiltrazione può essere facilmente incrementata, consentendo un buon controllo dei livelli di BUN anche negli stati di grave catabolismo. L'efficienza dell'emofiltrazione artero-venosa può essere aumentata anche implementando il sistema con componente diffusiva, effettuando quindi una emodialisi continua (CAVHD) (61) od una emodiafiltrazione continua $(62,63)$. Alcuni vantaggi tipici del trasporto convettivo verrebbero tuttavia perduti nell'emodialisi continua. Noi crediamo che il trattamento sopra descritto, denominato dialisi continua ad alto flusso (CHFD) (64) possa rappresentare il compromesso ottimale tra metodica pura diffusiva e metodica pura 
convettiva e che possa essere considerata un trattamento sostitutivo in terapia intensiva di prima scelta.

II rebound della concentrazione di soluti osservato alla fine della seduta dialitica nei trattamenti intermittenti non viene rilevato nei trattamenti continui, e ciò rappresenta un importante vantaggio per il controllo della concentrazione media di BUN nel tempo-e per una adeguata rimozione di urea. Infatti, mentre nell'emodialisi intermittente la concentrazione di BUN cala bruscamente dopo la prima ora di trattamento e l'estrazione di soluti diminuisce, nei trattamenti continui la concentrazione di BUN si mantiene pressoché costante, consentendo una rimozione relativamente elevata, soprattutto se comparata al valore di clearance. In Figura 4 sono riportate le clearance dell'urea e dell'inulina nei diversi tipi di trattamento. Si osservi come il miglior grado di depurazione ematica si ottenga con la nostra dialisi continua ad alto flusso, grazie al raggiungimento di un prodotto $\mathrm{Kt}$ (clearance $\mathrm{x}$ tempo) ottimale. L'efficienza complessiva di un trattamento dialitico dipende infatti dalle clearance ottenute, ma anche dalla durata del periodo per cui tali clearance sono applicate. II prodotto $\mathrm{Kt}$ esprime la quantità di acqua plasmatica depurata nel tempo t. Quando il tempo di trattamento è breve, come nella dialisi inter- mittente, per ottenere la stessa efficienza depurativa di un trattamento continuo a bassa clearance sono necessarie clearance superiori. In ogni caso, anche a parita di Kt la quantità totale di soluti rimossa sarà sempre maggiore nei trattamenti continui, per l'effetto di continuo refilling plasmatico di soluti dallo spazio intracellulare ed interstiziale e per il costante profilo di concentrazione plasmatica.

Pertanto i pazienti in dialisi intermittente, in confronto a quelli in terapia continua, si trovano per la maggior parte della giomata ad un maggiore livello di intossicazione uremica.

La CAVH e le terapie da essa derivate possono essere impiegate per correggere squilibri idroelettrolitici modificando la composizione del liquido di reinfusione. L'iponatremia e l'ipernatremia possono essere corrette non solo normalizzando la concentrazione plasmatica di sodio, ma anche ricostituendo un normale pool totale corporeo di sodio.

Anche l'iperkaliemia può essere corretta, ma l'efficienza del trattamento in questo caso è direttamente dipendente dalla quantità totale di ultrafiltrato e dalla sua sostituzione con soluzioni prive di potassio. L'efficienza della CAVH nel rimuovere potassio è piuttosto bassa e dipende dal livello di partenza della potassiemia. Per esempio, se la potassiemia

\section{CLEARANCE GIORNALIERE CON LE DIVERSE TECNICHE}



Fig. 4-Clearance espressa in litri/24 ore ontenata con diversi trattanicnti continui.

In dialisi continua ad alto flusso una clearance relativamente bassa applicata per 24 ore pud offrire una mi. gliore depurazione rispetto ad altri trattamenti ad elevata clearance applicata per 4 are al giorno.

iniziale è di $6 \mathrm{mEq} /$, una ultrafiltrazione di 10 litri elimina solo 60 milliequivalenti di potassio. D'altra parte, la stessa correzione dell' equilibrio acido-base consente un controllo più fisiologico del rapporto delle concentrazioni intra/extracellulari del potassio. Parallelamente vi è la possibilità di istituire un adeguato programma di supporto nutrizionale parenterale, che riduce il catabolismo promuovendo la captazione cellulare di glucosio e potassio. Non vi è dubbio comunque che la CAVHD sia molto più efficace in caso di iperkaliemia acuta. La perdita di bicarbonato durante CAVH può essere facilmente misurata direttamente nell'ultrafiltrato, o prevista secondo la formula:

$$
\mathrm{HCO}_{3}(\mathrm{f})=\mathrm{UF} \times \mathrm{HCO}_{3}(\mathrm{~s}) \times 1.124
$$

dove $\mathrm{HCO}_{3}(\mathrm{f})$ ed $\mathrm{HCO}_{3}(\mathrm{~s})$ sono le concentrazioni di bicarbonato nell 'ultrafiltrato e nel sangue rispettivamente; UF e I'ultrafiltrato totale e 1.124 è il coefficiente di sieving medio per il bicarbonato.

Quando la CAVH viene eseguita senza reinfusione, allo scopo di ridurre il sovraccarico di liquidi, le perdite di bicarbonato sono compensate dalla riduzione del volume di distribuzione corporeo per il tampone, la cui concentrazione ematica non varia significativamente. Al contrario, quando viene effettuata reinfusione per il mantenimento del bilancio di fluidi, per mantenere stabile la concentrazione ematica di $\mathrm{HCO}_{3}$ la perdita di bicarbonato deve essere compensata da somministrazione di pari quantità di tampone.

Infine, quando la CAVH viene utilizzata per la correzione dell'acidosi metabolica, la quantita di tampone reinfuso deve eccedere le perdite fino ad ottenere un bilancio positivo (65). In alcuni casi si puo utilizzare il lattato senza particolari problemi, valutando però attentamente il rischio di acidosi lattica.

Nella dialisi continua ad alto flusso (CHFD) il bicarbonato contenuto nel liquido di dialisí consente di mantenere un buon equilibrio di tamponi con piccole fluttuazioni e notevole stabilità clinica del paziente.

Può essere impiegato anche liquido di dialisi con acetato, ma $\mathrm{i}$ possibili effetti emodinamici collaterali od una ridotta velocità di metabolizzazione dell' acetato possono aumentare il rischio di intolleranza al trattamento. 


\section{Trattamenti sostitutivi continui nel neonato}

Una speciale indicazione per la CAVH è rappresentata dall'insufficienza renale acuta nei neonati e nei lattanti (66). L'uso di emofiltri di piccole dimensioni (Minifilter) e speciali circuiti consentono di eseguire tale trattamento in condizioni di sicurezza anche in questi pazienti.

La CAVH e la CAVHD rappresentano in questi casi una reale alternativa all'emodialisi od alla dialisi peritoneale, essendo generalmente ben tollerate. Vi cे stata una significativa evoluzione tecnologica negli ultimi 5 anni con lo sviluppo di nuovi minifiltri disegnati appositamente,

Ultrafiltrazioni di $1.2 \mathrm{ml} /$ minuto sono sufficienti per ottenere una adeguata depurazione ematica ed un buon bilancio di fluidi. Operativamente si possono ottenere flussi ematici di $20-50 \mathrm{ml} / \mathrm{min}$. mentre la richiesta di eparina è generalmente modesta per le basse frazioni di filtrazione che si ottengono nel filtro. I minifiltri contengono infatti un basso numero di fibre ad ampio diametro interno ( 570 micra) con una resistenza intrinseca molto bassa.

II trattamento può essere prolungato per più giorni o per settimane, fino al recupero della funzione renale, La CAVHe CAVHD rappresentano inoltre l'unica possibilità di istituire un programma di nutrizione parenterale in questi bambini, in cui spesso si osserva un grave stato catabolico.

\section{Complicanze dei trattamenti continui}

Le terapie sostitutive continue sono generalmente ben tollerate e presentano una bassa incidenza di complicanze. L'esito è correlato a diversi aspetti, tra cui la gravità dello stato clinico generale del paziente e la presenza o meno di ulteriori problematiche come la necessità di ventilazione meccanica o di supporto cardiovascolare artificiale.

Il numero di organi coinvolti nella sindrome di insufficienza multiorganica sembra essere di importanza critica non solo per il buon esito del trattamento, ma anche per la sopravvivenza del paziente. Il fatto che il tasso di mortalità non sia diminuito negli anni è verosimilmente dovuto alla sempre più frequente applicazione di queste strategie terapeutiche in pazienti con prognosi comunque molto grave,

Nella Tabella I sono riassunti $i$ dati relativi alla popolazione di pazienti (esclusivamente in terapia intensiva) con IRA complicata trattati nell'Ospedale di Vicenza dal 1982.

In base alla nostra esperienza te complicanze che si possono presentare nel corso dei trattamenti continui sono quelle elencate nella Tabella II.

Essendo l'emofiltrazione continua una tecnica invasiva, comporta di per sé alcuni peculiari fattori di rischio che devono essere preventivati. Le più gravi complicanze sono quelle inerenti all'accesso arterioso utilizzato in CAVH $(67,68)$.

TAB. I - DIAGNOSI E SOPRAVVIVENZA NELLA POPOLAZIONE TRATTATA

\begin{tabular}{lcc}
\hline Diagnosi & Trattati & Sopravvissuti \\
\hline Insufficienza Multiorganica & 18 & 5 \\
IRA da Politraumatismo & 25 & 15 \\
IRA Postchirurgica & 96 & 57 \\
Shock Emorragico & 12 & 7 \\
Insufficienza Cardiaca Congestizia & 17 & 15 \\
IRA nel Neonato & 18 & 9 \\
Miscellanea & 26 & 14 \\
Totale & 212 & 122 \\
\hline
\end{tabular}

L'impiego di metodiche con circolazione veno-venosa riduce questi rischi in modo rilevante. L'incannulazione percutanea con introduzione di cateteri di grosso calibro, secondo la metodica di Seldinger modificata, può comportare sanguinamenti ed espone al rischio di perforazione del vaso. Con una tecnica rigorosa ed in mani esperte ciò avviene molto raramente.

Tuttavia possono verificarsi importanti emorragie quando si operi su vasi la cui struttura sia alterata da lesioni aterosclerotiche, sia per fragilità della parete stessa che per accidentale distacco di un ateroma.

Un attento monitoraggio della coagulazione contribuisce a ridurre il rischio emorragico in emofiltrazione.

$\mathrm{La}$ rimozione del catetere, alla fine del trattamento, può comportare però comunque un sanguinamento, per cui si deve sempre effettuare una buona e persistente compressione del vaso. Se, nonostante questa manovra, il sanguinamento persiste, si deve ricorrere senza indugio all'emostasi chirurgica.

Le trombosi in sede di incannulazione arteriosa sono di non rara evenienza. e possono anche comportare grave ipoperfusione con ischemia dell'arto inferiore, nel qual caso è imperativo l'intervento chirurgico.

Pertanto è altamente raccomandabile un attento controllo della perfusione a valle dell'accesso arterioso $(69,70)$ nel corso del trattamento. Sebbene meno frequenti, tali complicanze possone verificarsi anche a carico dell accesso venoso.

Nei trattamenti in circolazione veno-venosa è d'obbligo l'impiego di una pompa ematica.

In caso di occlusione trombotica dell'accesso venoso di rientro, la pressione positiva all'interno del circuito ematico aumenta; tale anomalia, rilevata dai sistemi di servocontrollo della pompa, ne provoca l'arresto immediato. Non è raro comunque che quest'ultima evenienza si verifichi come conseguenza di una coagulazione massiva delle linee e del filtro, che, pur in assenza di ostruzioni trombotiche degli accessi vascolari o dei cateteri, produce all interno del circuito to stesso effetto.

La circolazione extracorporea comporta di per sé un elevato rischio di complicanze settiche. È molto importante quindi che la manipolazione del circuito extracorporeo avvenga sempre in condizio- 
TAB. II - COMPLICAZIONI DURANTE TERAPIE CONTINUE IN UN TOTALE DI 212 PAZIENTI (1982-1992)

\begin{tabular}{lcc}
\hline Complicazione & $\mathrm{n}$. & $\%$ \\
\hline Sanguinamento & 18 & 8.4 \\
Ematoma & 8 & 3.7 \\
Malfunzionamento dell'accesso & 1 & 0.4 \\
Sconnessione linee & 17 & 8.0 \\
Frequente coagulazione del filtro & 5 & 2.3 \\
Ipotensione secondaria al trattamento & 7 & 3.3 \\
Infezione del sito di incannulamento & 2 & 0.9 \\
Ipotermia & 4 & 1.9 \\
Ipofosfatemia & 5 & 2.3 \\
Trombosi venosa & 1 & 0.4 \\
Acidosi Lattica & 2 & 0.9 \\
Errore di bilancio di fluidi & 4 & 1.9 \\
Reazione anafilattica & - & - \\
Sindrome da deplezione & - & - \\
\hline
\end{tabular}

ni di massima sterilità. Il nostro atteggiamento in merito è di cambiare filtri e linee ogni 24 ore, anche se il sistema funziona correttamente, quale misura profilattica.

Nei pazienti in trattamento sostitutivo continuo la posologia degli antibiotici deve essere ovviamente adeguata alle condizioni cliniche ed alla clearance dialitica del farmaco. In molti casi, per mantenere costanti livelli terapeutici, le dosi e la frequenza di somministrazione devono essere aumentate, poiché il farmaco viene continuamente rimosso dal circolo attraverso l'emofiltro; per i farmaci ad alto legame proteico, la cui clearance è quindi molto bassa, la posologia deve essere invece ridotta rispetto a quella prevista per pazienti con normale funzione renale (71).

Qualsiasi accidentale sconnessione del circuito extracorporeo rappresenta un potenziale rischio letale. Tutte le connessioni devono pertanto essere controllate e ben fissate, e l'intero circuito deve essere bene in vista.

Per la maggior parte, gli episodi di sconnessione osservati nei nostri pazienti si sono verificati nei primi due anni di esperienza. All'epoca, peraltro, le linee ed i filtri non erano provvisti di connessioni di sicurezza "luer-lock", ed il circuito extracorporeo era ottenuto modificando manualmente le linee per emodialisi standard. Successivamente il proble- ma fu superato con i materiali specificamente progettati e costruiti dall'industria.

Nei moderni sistemi servoassistiti da pompa ematica l'embolia gassosa viene prevenuta da speciali dispositivi di mocontrollano l'arresto immediato della perfusione quando viene rilevata la presenza di aria nel circuito. Questi sistemi di sicurezza sono molto efficaci ed affidabili, eccetto in caso di difetti tecnici. Poiché la CAVH opera in condizioni di pressione positiva, l'evenienza di un risucchio gassoso nel circuito per pressione negativa distrettuale può verificarsi solo in corso di CVVH, in cui è previsto viene di solito in caso di connessioni difettose, di fissurazioni nella linea o di accidentali distrazioni durante infusione di liquidi nel circuito.

Un pericolo costantemente presente nei trattamenti continui è il sovraccarico di fluidi accidentali, in particolar modo quando lo schema terapeutico prevede un elevato ricambio di liquidi con grandi volumi di reinfusione. È quindi di massima importanza che nel corso del trattamento il bilancio di entrate ed uscite di liquidi sia attentamente monitorato e meticolosamente registrato, tenendo presente anche la possibilità di errori di trascrizione.

Raramente, per volumi di ultrafiltrazione nitoraggio e di allarme incorporati, che l'impiego di una pompa ematica. Ciò av- e reinfusione molto elevati, si è osservata la comparsa di ipotermia; questo inconveniente può essere comunque evitato con un semplice preriscaldamento dei liquidi da reinfondere, ma può essere anche sfruttato efficacemente proprio per abbassare la temperatura corporea in caso di ipertermia.

In pazienti sottoposti a trattamenti continui è stata osservata la comparsa di ipofosforemia.

Questa evenienza, come qualsiasi altro squilibrio elettrolitico, metabolico-nutrizionale o farmacologico, è evitabile con un attento monitoraggio dei parametri biochimici del paziente.

\section{Conclusioni}

Da queste considerazioni possiamo concludere che la CAVH ed i trattamenti da essa derivati possono rappresentare una terapia affidabile ed efficace per i pazienti critici con insufficienza renale acuta.

Semplicità, facilità di monitoraggio e di realizzazione rendono queste teciche di prima scelta nelle strategie di trattamento di molte condizioni cliniche.

Per pazienti con grave instabilità cardiovascolare, insufficienza multiorganica o politraumatizzati, $\mathrm{i}$ trattamenti sostitutivi continui offrono inoltre la possibilità di istituire un trattamento caratterizzato da alta tollerabilità ed effettiva "emoequilibrazione".

Sono oggi disponibili diverse tecniche che combinano diffusione e convezione in circolazione spontanea o con pompa ematica.

La scelta finale della tecnica e dei materiali dipende sempre comunque dalle necessità e dalle condizioni cliniche del paziente, dalle disponibilità della struttura ospedaliera e dalla preparazione ed addestramento del personale infermieristico e medico.

Le limitazioni classiche di bassa efficienza depurativa o di frequente coagulazione dei filtri sono state superate, mentre sono state proposte nuove indicazioni e prospettati specifici vantaggi in diversi quadri clinici.

Per tutti questi motivi è lecito pensare che, in futuro, la CAVH e le altre terapie sostitutive continue derivate saranno sempre più impiegate nel trattamento dei pazienti con grave compromissione cli- 


\section{BIBLIOGRAFIA}

1. Knochel J. Biochemical, electrolyte and acid-base disturbances in acute renal failure. In: Brenner $B M$ and Lazarus JM, eds. Acute Renal Failure. Philadelphia: W.B. Saunders, Co., 1983; 568-85.

2. Lien J, Chan V. Risk factors influencing survival in acute renal failure treated by hemodialysis. Arch Intern Med 1985; 145: 2067.

3. Henderson LW, Besarab A, Michaels A, Bluemle LW Jr. Blood purification by ultrafiltration and fluid replacement (diafiltration). Trans ASAIO 1967; 17: 216-21.

4. Silverstein ME, Ford CA. Lysaght MT, Henderson LW. Treatment of severe fluid overload by ultrafiltration. New Engl J Med $1974 ; 291: 747$.

5. Kramer P, Wigger W, Rieger J. Matthaei D, Scheler F. Arteriovenous hemofiltration: a new and simple method for treatment of overhydrated patients resistant to diuretics. Klin Wschr 1977; 55 : 1121.

6. Lauer A, Saccaggi A, Ronco C, Belledonne M, Glabman S, Bosch JP. Continuous arteriovenous hemofiltration in the critically ill patient. Ann Intern Med 1983: 99: 455.

7. Ronco C, Brendolan A, Bragantini L, et al. Continuous arteriovenous hemofiltration. Contr Nephrol 1985;48: 70-8.

8. Bartlett R, Bosch JP, Paganini EA, Geronemus R, Ronco C. Continuous arteriovenous hemofiltration. Trans ASAIO 1987; 38 : 345.

9. Paganini EP. Acute Continuous Renal Replacement Therapy. Boston: Martinus Nijhoff Publishing. 1986.

10. Olbricht CJ, Schurek HJ. Tytul S, Muller C, Stolte H. Comparison between Scribner shunt and femoral catheters as vascular access for continuous arteriovenous hemofiltration. In: Kramer P, ed. Arteriovenous hemofiltration. Berlin: Springer Verlag, 1985; 57-66.

11. Olbricht CJ, Schurek HJ, Stolte $\mathrm{H}$, Koch KM. The influence of vascular access modes on the efficiency of CAVH. In: Sieberth HG. Mann $\mathrm{H}$, eds. Continuous Arteriovenous Hemolfiltration. Basel: Karger 1985; 14-24.

12. La Greca G, Fabris A, Ronco $\mathrm{C}$. CAVH Proceedings of the International Symposium on Continuous Arteriovenous Hemofiltration. Vicenza 1986. Milano: Wichtig Editore, 1986.

13. Ronco $C$, Brendolan $A$, Bragantini L, et al. Self limited dehydration during CAVH. Blood Purif $1984 ; 2: 88$.

14. Ronco $\mathrm{C}$, Brendolan $\mathrm{A}$, Bragantini L, et al. Studies on blood flow dinamic and ultrafiltration $\mathrm{ki}$ netics during continuous arteriovenous hemofiltration. Blood Purif $1986 ; 4: 220$.

15. Henderson LW. Biophysics of ultrafiltration and hemofiltration. In: Replacement of renal function by dialysis. Ed, J.F. Maher, Kluwer Academic Publ,., Dordrecht 1989; 300-26.

16. Ronco C, Bosch JP, Lew S, et al. Technical and clinical evaluation of a new hemofilter for CAVH; Theoretical concepts and practical applications of a different blood flow geometry, In: La Greca G, Fabris A, Ronco C, eds. Proceedings of the International Symposium on Continuous Arteriovenous Hemofiltration on CAVH, Vicenza 1986. Milano: Wichtig Editore 1986: 55-61.

17. Golper TA, Ronco C, Kaplan AA. Continuous arterio-venous hemofiltration: Improvements, Modifications and future directions. Seminars in Dialysis 1988; 1: 50-4.

18. Pallone TL, Peterson $\mathrm{J}$. Continuous arteriovenous hemofiltration, an in vivo simulation. Trans
ASAIO 1987: 33: 304-8.

19. Ronco C, Brendolan A, Bragantini $\mathrm{L}$, et al. Continuous arteriovenous hemofiltration with AN69S membrane; procedures and experience. Kidney Int 1988; 33 (suppl 24): 150-3.

20. Kaplan A, Longnecker RE. Folkert VW. Continuous arteriovenous hemofiltration. Ann Intern Med 1984; 100: 358-64.

21. Ronco C, Brendotan A, Bragantini L, et al. Technical and clinical evaluation of a new polyamide hollow fiber hemofilter for CAVH. Int J Artif Organs 1988: 11: 33-8.

22. Lysaght MJ, Schmidt B, Gurland HJ. Mass Transfer in arteriovenous hemofiltration. In: Kramer $P$, ed. Arteriovenous Hemofiltration. Berlin: SpringerVerlag, 1985; 3-13.

23. Lysaght MJ. Hemodialysis membranes in transition. Contribution to Nephrology, Basel: Karger Verlag, 1988; 61: 1-17.

24. Lysaght MJ. Boggs D, Transport in continuous arteriovenous hemofiltration and slow continuous uifrafiltration. In: Paganini E, ed. Acute Continuous Renal Replacement Therapy. Boston: MartinusNijhof, 1986; 43-50.

25. Kaplan AA. Predilution vs postdilution for continuous arteriovenous hemofiltration. Trans Am Soc Artif Intern Organs 1985; 31: 28-31.

26. Kaplan AA. The effect of predilution during continuous arteriovenous hemofiltration (Abstract). Am Soc Nephrol 1984: 66A.

27. Golper TA, Kaplan AA, Narasimhan N, Leone M. Transmembrane pressures generated by filtrate line suction maneuvers and predilution fluid replacement during in vitro continuous arteriovenous hemofiltration. Int J Artif Organs 1987: 10: 41-6.

28. Kaplan AA. Clinical trials with predilution and vacuum suction: 
Enhancing the efficiency of the CAVH treatment. Trans Am Soc Artif Intern Organs 1986: 32: 49-51.

Kaplan AA, Longnecker RE, Folkert VW. Suction assisted continuous arterio-venous hemofiltration. Trans Am Soc Artif Intern Organs, 1983: 29: 408-12.

30. Ronco C. Brendolan A. Bragantini L. et al. Arteriovenous hemodiafiltration associated with continuous arteriovenous hemofiltration. A combined therapy in the hypercatabolic patients. In: La Greca G. Fabris A. Ronco C. eds. Proceedings of the International Symposium on Continuous Arteriovenous Hemofiltration on $\mathrm{CAVH}$ Vicenza 1986. Milano: Wichtig Editore 1986: 171-83.

31. Geronemus R, Schneider N. Continuous arterio-venous hemodialysis: a new modality for treatment of acute renal failure. Trans ASAIO 1984; XXX: 610-3.

32. Paganini EP, Fouad F, Tarazi RC, Bravo EL, Nakamoto $\mathrm{S}$. Hemodynamics of isolated ultrafiltration in chronic hemodialysis pattients. Trans ASAIO 1979: XXV: 422-5.

33. Geronemus R, Schneider N. Continuous Arteriovenous hemo. dialysis. In: Paganini E. Geronemus $R$, eds. Proceedings of the Third International Symposium on Acute Continuous Renal Replacement Therapy. Ft. Lauderdale, 1987: 77.

34. Ronco C, Brendolan A Bragantini L. et al. Solute and Water transport during Continuous arteriovenous hemofiltration. Int J Artif Organs 1987; 10: 179-84.

35. Ronco C. Arterio-venous hemodiafiltration (AVHDF): a possible way to increase urea removal during CAVH. Int J Artif Organs 1985: 8:61-2.

36. Ronco C. Burchardi $H$. Management of acute renal failure in intensive care patients. In: Pathophysiologic foundations of critical care. Ed. JF Dhainaut, M. Pinsky, Williams \& Wilkins,
Baltimore 1992, Chapter 38: 630-76.

37. Ronco C. Continuous arteriovenous hemofiltration: optimization of technical procedures and new directions. In: New perspectives in hemodialysis, peritoneal dialysis, arteriovenous hemofiltration and plasmapheresis. WH Horl and P Schollmeyer Eds. Plenum Press, New York. 1989: 167.

38. Graziani G, Casati S, Ponticelli C. CAVH e CAVHD nel paziente acuto ipercatabolico. Nefrologia Dialisi e Trapianto. 1986: 31:235.

39. Olbricht CJ, Hebel U, Frei U, Koch KM. High performance CAVH without pumps, suction and predilution. Blood Purif 1986; 4: 222.

40. Zobel G, Trop M, Muntean W, Ring E, Gleispach H. Anticoagulation for Continuous Arteriovenous Hemofiltration in children. Blood Purif 1988; 6: 90-5.

41. Maher JF, Lapierre L. Schreiner GE, Geoger M. Westervelt FB. Regional heparinization for hemodialysis. N Engl 3 Med 1963: 268: 451 .

42. Pinnick RV, Wiegmann TB, Diederich DA. Regional citrate anticoagulation for hemodialysis in patients at high risk for bleeding. $\mathrm{N}$ Engl J Med 1983: 308:258.

43. Zusman RM, Rubin RH, Cato A. Hemodialysis using prostacyclin instead of heparin as the sole antithrombotic agent. N Engl J Med 1981; 304: 934 .

44. Josefowicz M, Josefowicz J. New approaches to anticoagulation with heparin-like biomaterials. ASAIO J 1985: 8: 218 .

45. Hirsch J, Ofosu F, Buchanan M. Rationale behind the development of low molecular weight heparin derivates. Semin Thromb Hemost 1985: 11: 13.

46. Kramer P. Schrader J, Bohnsack W, Grieben G. Grone HJ, Scheler F. Continuous arteriovenous hemofiltration: A new kidney repla- cement therapy. Proc EDTA 1981: 18: 743 .

47. Lysaght MJ, Boggs D. Transport in continuous arteriovenous hemofiltration and slow continuous ultrafiltration. In: Paganini E, ed. Acute Continuous Renal Replacement Therapy. Boston: Martinus Nijhof, 1986: 43-50.

48. Golper TA, Ronco C. Kaplan AA. Continuous arterio-venous hemofiltration: Improvements, Modifications and future directions. Seminars in Dialysis, 1988: 1: 50-4.

49. Olbricht C, Mueller C, Schurek HJ. Treatment of acute renal failure in patients with multiple organ failure by continuous spontaneous hemofiltration. Trans ASAIO 1982; 28: 33-7.

50. Kramer P, Bhler J, Kehr A, et al. Intensive care potential of continuous arteriovenous hemodialysis. Trans ASAIO 1982; 28: 28-32.

51. Bosch JP. Ronco C. Continuous arteriovenous hemofiltration. In: Replacement of renal function by dialysis, JF Maher Ed. Kluwer Academic Publ. Dordrecht 1989; 15: $347-61$.

52. Lauer A, Alvis R, Beal A, Avram M. Hemodynamic consequences of continuous arteriovenous hemofiltration in intractable fluid overload (abstract). Kidney Int 1986; $29: 218$.

53. Burchardi H. Die kontinuierliche arterio-venose Hemofiltration (CAVH). Intensivmed Notfallmed Anesthes 1987; 62:51-9.

54. Knaus WA, Draper EA, Wagner DP, et al. APACHE II - A severity of disease classification. Crit Care Med 1985; 13: 818-22.

55. Little RA, Campbell IT, Green CJ, Kishen R, Waldek S. Nutritional support in acute renal failure in the critically ill. In: Bihari D, Neild G (eds) Acute renal failure in the intensive therapy unit. Springer Verlag, Heidelberg 1990; 347-56.

56. Bellomo R, Tipping P, Boyce 
N. Tumor necrosing factor clearances during veno-venous hemodiafiltration in the critically ill. Trans Am Soc Artif Int Organs, 1991; 37: 322-3.

57. deVries I, van Deventer SJH, Debetes J. Endotoxin induced cytokines in human septicemia. Adv. Exp Med Biol 1990; 256: 635-40.

58. Golper TA. Continuous arteriovenous hemofiltration in acute renal failure. Am J Kidney Dis 1985; 6: 373-8.

59. Geronemus R, von Albertini B, Glabman S, Lysaght M, Kahn T, Bosch JP. Enhanced molecular clearance in hemofiltration. Proc Clin Dial Transplant Forum 1978; 8: 147-52.

60. Sigler M, Teehan BP. Solute transport in slow continuous arteriovenous hemodialysis: An improved method for treating acute renal failure. Proc Third Int Symposium on Acute Continuous Renal Replacement Therapy, Fort Lauerdale, FL 1987; 3: 78-85.

61. Geronemus R, Schneider N. Continuous arteriovenous hemodialysis: A new modality for treatment of acute renal failure. Trans ASAIO 1984; 30: 610-15.

62. Ronco C. Arteriovenous hemodiafiltration (AVHDF): a possible way to increase urea removal during CAVH. Int J Artif Organs, 1985; 8: 61-2.

63. Ronco C, Brendolan A. Bragantini L, Arteriovenous hemodiafiltration associated with continuous arteriovenous hemofiltration. A combined therapy in the hypercatabolic patient. In: Proceedings of the International Symposium on Continuous arteriovenous hemofiltration on CAVH, Vicenza 1986 , La Greca G, Fabris A, Ronco C, Eds, Wichtig Editore Milano, 1986; 171-83.

64. Ronco C, Burchardi $\mathrm{H}$. Management of acute renal failure in the critically ill patient. In: Pathophysiologic foundation of critical care. Ed. Pinsky M, Dhainaut
JF, William \& Wilkins, Baltimore. 1992, Chapter 38: 630-76.

65. Raimondi F, Bianchi T, Emmi $\mathrm{V}$; Use of continuous arteriovenous hemofiltration $(\mathrm{CAVH})$ in lactic acidosis: A case report. In: La Greca G. Fabris A, Ronco C, eds. Proceedings of the International Symposium on Continuous Arteriovenous Hemofiltration on CAVH, Vicenza. Milano: Wichtig Editore, 1986; 135-40.

66. Ronco C, Brendolan A, Borin D, et al. Continuous arteriovenous hemofiltration in newborns. Int Conference CAVH Aachen, Karger. Basel, 1984; 76-9.

67. Kramer P. Limitations and pitfalls of continuous arteriovenous hemofiltration. In: Kramer P (ed): Arteriovenous Hemofiltration. Springer Verlag, Heidelberg 1985; 206-11.

68. Stokke T, Kettler D. Komplikationen der Kontinuierlichen arteriovenos en Hemofiltration. Anaesthesist 1985; 34: 528-33.

69. Sheretz RJ, Falk RJ, Huffman KA, Thoman CA. Mattern WD. Infections associated with subclavian Uldall catheters. Arch Intern Med 1983; 143: 52-7.

70. Rozsival V, Bastecka D, Fixa PO, Kozak J, Herout V. Lont term experience with the technique of subclavian and femoral vein cannulation in hemodialysis. Artif. Organs, 1979; $3: 241-7$. 